\title{
Ambiguities in the local thermal behavior of the scalar radiation in one-dimensional boxes
}

\author{
E. S. Moreira, Jr. \\ Instituto de Matemática e Computação, Universidade Federal de Itajubá, \\ Itajubá, Minas Gerais 37500-903, Brazil
}

(Received 30 May 2020; accepted 24 September 2020; published 26 October 2020)

\begin{abstract}
In this paper we report on certain ambiguities in the calculation of the ensemble average $\left\langle T_{\mu \nu}\right\rangle$ of the stressenergy-momentum tensor of an arbitrarily coupled massless scalar field in one-dimensional boxes in flat spacetime. The study addresses a box with periodic boundary conditions (a circle) and boxes with reflecting edges (with Dirichlet or Neumann boundary conditions at the end points). The expressions for $\left\langle T^{\mu \nu}\right\rangle$ are obtained from finite-temperature Green functions. In an appendix, in order to control divergences typical of two dimensions, these Green functions are calculated for related backgrounds with an arbitrary number of dimensions and for scalar fields of arbitrary mass, and are specialized in the text to two dimensions and for massless fields. The ambiguities arise due to the presence in $\left\langle T^{\mu \nu}\right\rangle$ of double series that are not absolutely convergent. The order in which the two associated summations are evaluated matters, leading to two different thermodynamics for each type of box. In the case of a circle, it is shown that the ambiguity corresponds to the classic controversy in the literature whether or not zero-mode contributions should be taken into account in computations of partition functions. In the case of boxes with reflecting edges, it results that one of the thermodynamics corresponds to a total energy (obtained by integrating the nonhomogeneous energy density over space) that does not depend on the curvature coupling parameter $\xi$ as expected, whereas the other thermodynamics curiously corresponds to a total energy that does depend on $\xi$. Thermodynamic requirements (such as local and global stability) and their restrictions on the values of $\xi$ are considered.
\end{abstract}

DOI: 10.1103/PhysRevD.102.085014

\section{INTRODUCTION}

Over the last decades, since the discovery that a black hole behaves very much like a blackbody-with entropy and temperature [1], and even radiation [2] — the study of quantum fields at finite temperature near boundaries and in spacetimes with nontrivial topologies and geometries has received increasing attention in the literature. The message seems to be that one may learn a great deal about the nature of gravity itself by looking at boundary quantum field theory at finite temperature, especially in lower dimensions as holography suggests [3].

A simple example that is commonly used to illustrate the interplay between thermodynamics of fields and nontrivial topology is the model of a massless scalar field living on a circle of length $a$ and geometry (throughout the text $k_{B}=\hbar=c=1$ )

\footnotetext{
*moreira@unifei.edu.br
}

Published by the American Physical Society under the terms of the Creative Commons Attribution 4.0 International license. Further distribution of this work must maintain attribution to the author(s) and the published article's title, journal citation, and DOI. Funded by SCOAP.

$$
d s^{2}=d t^{2}-d x^{2}
$$

Familiar methods in statistical mechanics lead to the internal energy,

$$
U(T, a)=-\frac{\pi}{6 a}+\frac{4 \pi}{a} \sum_{k=1}^{\infty} \frac{k}{e^{2 \pi k / T a}-1},
$$

where the first term is the vacuum energy (i.e., corresponding to $T \rightarrow 0$ ) [4] and the second is the contribution at temperature $T$ due to the Planck distribution [5]. In fact, Eq. (2) disguises a subtlety that has simply been ignored. It turns out that due to the proper periodic boundary condition of a circle, in evaluating $U$ one should also take into account the mode corresponding to $k=0$, i.e., the so-called "zero mode" [6]. However, in order to do so some regularization must be used. For example, the term ${ }^{1}$

$$
\frac{2 \pi}{a} \lim _{\epsilon \rightarrow 0} \frac{\epsilon}{e^{2 \pi \epsilon / T a}-1}
$$

\footnotetext{
${ }^{1}$ Note the factor $2 \pi / a$ in Eq. (3), and not $4 \pi / a$ as in Eq. (2) where each term in the summation corresponds to two states.
} 
could be added to Eq. (2), resulting in

$$
U(T, a)=-\frac{\pi}{6 a}+T+\frac{4 \pi}{a} \sum_{k=1}^{\infty} \frac{k}{e^{2 \pi k / T a}-1} .
$$

The contrasting expressions in Eqs. (2) and (4) are the source of a dispute in the literature regarding whether the zero mode should be taken into account or not [7-9]. This issue is relevant since it is related to the third law of thermodynamics $[6,7,9]$, the derivation of the CardyVerlinde formula, and entropy bounds [10,11], among other topics [12].

Arguing in favor of Eq. (2), the authors of Ref. [7] remarked that an independent calculation using the thermal Green function leads to a homogeneous energy density $\left\langle T_{t t}\right\rangle$ which, multiplied by the length $a$, yields precisely $U$ in Eq. (2). Indeed, when looking through the literature one finds an earlier calculation in a textbook [4], where the ensemble average $\left\langle T_{\mu \nu}\right\rangle$ of the stress-energy-momentum tensor is determined from the thermal Green function. Then, by taking $\left\langle T_{t t}\right\rangle \times a$, Eq. (2) comes up again. It is rather puzzling that such a "local approach" to obtain the internal energy ignores the term $T$ in Eq. (4).

The apparent absence of Eq. (4) in the "local approach" has motivated the investigation in Sec. II, whose content is now outlined. In Appendix A 1, the finite-temperature Green function for a neutral scalar field of mass $M$ in a flat $N$-dimensional spacetime with periodic boundary condition along one of the dimensions is calculated. By taking $M \rightarrow 0$ and $N \rightarrow 2$, the Green function is used in Sec. II A to obtain $\left\langle T_{\mu \nu}\right\rangle$ of a massless scalar field on a circle of length $a$. As is typical in this kind of calculation involving finite-temperature Green functions to obtain the ensemble average of the stress-energy-momentum tensor [13-16], the resulting expression for the homogeneous $\left\langle T_{\mu \nu}\right\rangle$ contains a double series. In higher dimensions the double series converges absolutely, meaning that one can interchange the order of the summations and the result will still come out the same (see, e.g., Ref. [17]). However, this is not the case in a two-dimensional background, as the calculations in Sec. II show: evaluating the summations at one order leads indeed to Eq. (2), but interchanging the order of the summations leads instead to Eq. (4).

In Sec. II B the local thermodynamic stability of the two sides of the ambiguity is addressed. In Sec. II C, in order to further explore the implications of this ambiguity in thermodynamics, the formula (with $\beta:=1 / T$ )

$$
U=\left(\frac{\partial(\beta F)}{\partial \beta}\right)_{a}
$$

is integrated to determine the Helmholtz free energy $F$. The integration constant resulting from this procedure is set by requiring that the thermodynamic pressure matches the stress component of $\left\langle T_{\mu \nu}\right\rangle$, such that there is no unknown length scale. Various thermodynamics aspects are investigated at the asymptotic limits when $T a \ll 1$ and when $T a \gg 1$.

It is rather well known that an interval with the Neumann boundary condition at the end points is a model of a onedimensional box with reflecting edges, in which the calculation of the partition function for hot scalar radiation also leads to a zero mode commonly ignored. In fact, the formula given in the literature for the internal energy corresponding to the Dirichlet boundary condition at the end points (for which there is no zero mode) is the same as that corresponding to the Neumann boundary condition, namely $[18,19]$,

$$
U(T, a)=-\frac{\pi}{24 a}+\frac{\pi}{a} \sum_{k=1}^{\infty} \frac{k}{e^{\pi k / T a}-1},
$$

where $a$ is the length of the interval. Considering this fact and the discussion in the previous paragraphs, one may wonder if the local approach using Green functions may contain surprises here as well. When examining the literature, we have not found any study of $\left\langle T_{\mu \nu}\right\rangle$ for hot scalar radiation with Dirichlet or Neumann boundary conditions at the end points of a one-dimensional box. ${ }^{2}$ Such a study is implemented in Sec. III A and outlined below.

In Appendix A 2, the finite-temperature Green function for a neutral scalar field of mass $M$ in $N$-dimensional flat spacetime with two parallel plane walls at which either Dirichlet or Neumann boundary conditions are taken is evaluated. Then, in Sec. III A we again set $M \rightarrow 0$ and $N \rightarrow 2$ and use the Green function to obtain $\left\langle T_{\mu \nu}\right\rangle$ for a massless scalar field in a one-dimensional box of length $a$ with reflecting end points. This time, it turns out that $\left\langle T_{\mu \nu}\right\rangle$ is nonhomogeneous and dependent on the curvature coupling parameter $\xi$. Ambiguities now arise due to the presence in $\left\langle T_{\mu \nu}\right\rangle$ of two sets of double series. A particular order of summation is chosen in each series, and then the order is interchanged, resulting in two different expressions for $\left\langle T_{\mu \nu}\right\rangle$. The corresponding local thermal behaviors are investigated near the end points and at the midpoint of the box, for low and high temperatures. The values of $\xi$ consistent with local stable thermodynamic equilibrium (see Ref. [20]) are determined in Sec. III B.

In Sec. III C, in order to calculate the internal energies corresponding to the two expressions for $\left\langle T_{\mu \nu}\right\rangle$ found in Sec. III A, we integrate the nonhomogeneous energy densities $\left\langle T_{t t}\right\rangle$ over the box. It is then shown that one of the integrations leads to the formula in the literature (calculated using the partition function), i.e., Eq. (6), whereas the other integration instead yields

\footnotetext{
${ }^{2}$ In fact, there is such a calculation in four dimensions [16], but then there is no ambiguity since the corresponding double series are absolutely convergent.
} 


$$
U(T, a)=-\frac{\pi}{24 a}+(1 \mp 4 \xi) \frac{T}{2}+\frac{\pi}{a} \sum_{k=1}^{\infty} \frac{k}{e^{\pi k / T a}-1},
$$

where (as in the rest of the text) the upper sign applies to the Dirichlet boundary condition and the lower sign to the Neumann boundary condition. It is worth noting that Eqs. (2) and (4) correspond to Eqs. (6) and (7), respectively.

The appearance of $\xi$ in Eq. (7) is a bit surprising since, for a massless scalar field $\phi$ in flat spacetime with one spatial dimension $x$, the classical expression for the energy density $T_{t t}$ depends on the curvature coupling parameter $\xi$ only through the term $-2 \xi \partial_{x}\left(\phi \partial_{x} \phi\right)$, which thus does not contribute when integrating $T_{t t}$ for Dirichlet or Neumann boundary conditions [21]. It should also be noticed that by setting $\xi=1 / 4$ in Eq. (7), the Neumann zero mode mentioned above emerges. A last inescapable remark on Eq. (7) at this early stage in the paper involves the lowtemperature behavior of the corresponding heat capacity at constant volume, namely, $C=(1 \mp 4 \xi) / 2$ (up to a positive exponentially small correction). As thermal stability requires $C>0$ [22], it follows that $\xi$ must be such that $\xi \leq 1 / 4$ and $\xi \geq-1 / 4$ for Dirichlet and Neumann boundary conditions, respectively. Later on in the text, these inequalities will be confronted with those obtained in Sec. III B where local stable thermodynamic equilibrium is required [20].

The rest of the material in Sec. III C further investigates the two thermodynamics corresponding to Eqs. (6) and (7). Again, Eq. (5) is used to obtain $F$, and from that the other thermodynamic quantities whose behaviors are studied when $T a \ll 1$ and when $T a \gg 1$. As in Sec. II C, various thermodynamic aspects are addressed. It should be mentioned that, unlike the other cases, requiring that the thermodynamic pressure be equal to the stress component of $\left\langle T_{\mu \nu}\right\rangle$ corresponding to Eq. (7) gives rise to a length scale.

Section IV contains a summary and further discussion of the results.

\section{CIRCLE}

In this section we consider the thermal behavior of a massless scalar field living on a circle of length $a$. Therefore, the geometry is that in Eq. (1) and the end points $x=0$ and $x=a$ are identified.

\section{A. $\left\langle\boldsymbol{T}_{\boldsymbol{\mu \nu}}\right\rangle$}

The ensemble average $\left\langle T_{\mu \nu}\right\rangle$ can be formally obtained by using the "point splitting" method with the Feynman propagator $G_{\mathcal{F}}\left(\mathrm{x}, \mathrm{x}^{\prime}\right)$ at finite temperature $T=1 / \beta$ (see, e.g., Refs. [4,23] or the short review in Ref. [24]). In a flat background,

$$
\begin{aligned}
\left\langle T_{\mu \nu}\right\rangle= & i \lim _{\mathrm{x}^{\prime} \rightarrow \mathrm{x}}\left[(1-2 \xi) \nabla_{\mu} \nabla_{\nu^{\prime}}+(2 \xi-1 / 2) g_{\mu \nu} \nabla_{\sigma} \nabla^{\sigma^{\prime}}\right. \\
& \left.-2 \xi \nabla_{\mu} \nabla_{\nu}\right] G_{\mathcal{F}}\left(\mathrm{x}, \mathrm{x}^{\prime}\right)
\end{aligned}
$$

where $G_{\mathcal{F}}\left(\mathrm{x}, \mathrm{x}^{\prime}\right)$ is related to the Green functions calculated in the Appendix by

$$
G_{E}\left(\mathrm{x}, \mathrm{x}^{\prime}\right)=i G_{\mathcal{F}}\left(\mathrm{x}, \mathrm{x}^{\prime}\right) .
$$

For the analysis that will be implemented below, it is convenient to express each component of $\left\langle T_{\mu \nu}\right\rangle$ explicitly. It follows that the energy density is given by

$\left\langle T_{t t}\right\rangle=\frac{i}{2} \lim _{\mathrm{x}^{\prime} \rightarrow \mathrm{x}}\left[\frac{\partial^{2}}{\partial t \partial t^{\prime}}+(1-4 \xi) \frac{\partial^{2}}{\partial x \partial x^{\prime}}-4 \xi \frac{\partial^{2}}{\partial t^{2}}\right] G_{\mathcal{F}}\left(\mathrm{x}, \mathrm{x}^{\prime}\right)$,

the stress by

$$
\left\langle T_{x x}\right\rangle=\frac{i}{2} \lim _{\mathrm{x}^{\prime} \rightarrow \mathrm{x}}\left[\frac{\partial^{2}}{\partial x \partial x^{\prime}}+(1-4 \xi) \frac{\partial^{2}}{\partial t \partial t^{\prime}}-4 \xi \frac{\partial^{2}}{\partial x^{2}}\right] G_{\mathcal{F}}\left(\mathrm{x}, \mathrm{x}^{\prime}\right),
$$

and the fluxes by

$$
\left\langle T_{t x}\right\rangle=i \lim _{\mathrm{x}^{\prime} \rightarrow \mathrm{x}}\left[(1-2 \xi) \frac{\partial^{2}}{\partial t \partial x^{\prime}}-2 \xi \frac{\partial^{2}}{\partial t \partial x}\right] G_{\mathcal{F}}\left(\mathrm{x}, \mathrm{x}^{\prime}\right)
$$

and

$$
\left\langle T_{x t}\right\rangle=i \lim _{\mathrm{x}^{\prime} \rightarrow \mathrm{x}}\left[(1-2 \xi) \frac{\partial^{2}}{\partial x \partial t^{\prime}}-2 \xi \frac{\partial^{2}}{\partial x \partial t}\right] G_{\mathcal{F}}\left(\mathrm{x}, \mathrm{x}^{\prime}\right) .
$$

The reason for the appearance of the curvature coupling parameter $\xi$ in Eqs. (9)-(12), in spite of the flat geometry in Eq. (1), is that $T_{\mu \nu}$ is defined by a functional derivative with respect to an arbitrary metric [4].

Considering Eqs. (8), (A12), and (A13), one has that (keeping for the time being $N$ and $M$ arbitrary such that divergences can be properly controlled)

$$
G_{\mathcal{F}}\left(\mathrm{x}, \mathrm{x}^{\prime}\right)=\sum_{m=-\infty}^{\infty} \sum_{n=-\infty}^{\infty} f\left(\sigma^{(m, n)}\right)
$$

where

$$
f(u):=-\frac{i}{(2 \pi)^{N / 2}} M^{\frac{N-2}{2}}(-u)^{\frac{2-N}{4}} K_{\frac{N-2}{2}}(M \sqrt{-u}) .
$$

It is convenient to break the expression in Eq. (13) into the following contributions: 


$$
\begin{gathered}
G_{0}\left(\mathrm{x}, \mathrm{x}^{\prime}\right):=f\left(\sigma^{(0,0)}\right), \\
G_{\text {vacuum }}\left(\mathrm{x}, \mathrm{x}^{\prime}\right):=\sum_{n=-\infty}^{\infty} f\left(\sigma^{(0, n)}\right), \\
G_{\text {thermal }}\left(\mathrm{x}, \mathrm{x}^{\prime}\right):=\sum_{m=-\infty}^{\infty} f\left(\sigma^{(m, 0)}\right), \\
G_{\text {mixed }}\left(\mathrm{x}, \mathrm{x}^{\prime}\right):=\sum_{m=-\infty}^{\infty} \sum_{n=-\infty}^{\infty} f\left(\sigma^{(m, n)}\right),
\end{gathered}
$$

with a prime in the summation indicating that the term corresponding to $m=0$ or $n=0$ should be excluded. Noting Eqs. (13) and (14), and considering the asymptotic behavior of $K_{\nu}(\mathrm{z})$ [25], one sees that $G_{0}\left(\mathrm{x}, \mathrm{x}^{\prime}\right)$ is the familiar vacuum propagator in Minkowski spacetime, $G_{\text {vacuum }}\left(\mathrm{x}, \mathrm{x}^{\prime}\right)$ is the vacuum propagator due to a finite length $a$ (which vanishes if $a \rightarrow \infty), G_{\text {thermal }}\left(\mathrm{x}, \mathrm{x}^{\prime}\right)$ is the familiar thermal propagator in Minkowski spacetime (corresponding to the Planck distribution, and thus vanishing if $T \rightarrow 0$ ), and that $G_{\text {mixed }}\left(\mathrm{x}, \mathrm{x}^{\prime}\right)$ has a "mixed" nature (vanishing if $a \rightarrow \infty$ or if $T \rightarrow 0$ ). Since the background is flat, renormalization is implemented by dropping $G_{0}\left(\mathrm{x}, \mathrm{x}^{\prime}\right)$, yielding the renormalized propagator $G\left(\mathrm{x}, \mathrm{x}^{\prime}\right)$ that will replace $G_{\mathcal{F}}\left(\mathrm{x}, \mathrm{x}^{\prime}\right)$ in Eqs. (9)-(12),

$$
G\left(\mathrm{x}, \mathrm{x}^{\prime}\right)=G_{\text {vacuum }}\left(\mathrm{x}, \mathrm{x}^{\prime}\right)+G_{\text {mixed }}\left(\mathrm{x}, \mathrm{x}^{\prime}\right)+G_{\text {thermal }}\left(\mathrm{x}, \mathrm{x}^{\prime}\right) .
$$

Setting $N=2$ in Eq. (14), when $M \rightarrow 0$ it follows that [25]

$f(u)=\frac{i}{2 \pi}\left[\ln \left(\frac{M \sqrt{-u}}{2}\right)+\gamma\right]+\cdots, \quad N=2$.

Differentiating Eq. (18) and then setting $M=0$ gives

$$
f^{\prime}(u)=\frac{i}{4 \pi u},
$$

which is used in Eqs. (9)-(12) to determine the components of $\left\langle T_{\mu \nu}\right\rangle$ when $N=2$ and $M=0$. [Clearly, for $N=2$, only the two first terms in Eq. (A13) are taken into account.] The action of the differential operators in Eqs. (9)-(12) on Eq. (17) yields

$$
\left\langle T_{\mu \nu}\right\rangle=\left\langle T_{\mu \nu}\right\rangle_{\text {vacuum }}+\left\langle T_{\mu \nu}\right\rangle_{\text {mixed }}+\left\langle T_{\mu \nu}\right\rangle_{\text {thermal }} .
$$

Each contribution in Eq. (20) is diagonal with the energy densities equaling the corresponding stresses, which leads to

$$
\left\langle T_{x x}\right\rangle=\left\langle T_{t t}\right\rangle, \quad\left\langle T_{t x}\right\rangle=\left\langle T_{x t}\right\rangle=0 .
$$

According to Eq. (9),

$$
\left\langle T_{t t}\right\rangle=\left\langle T_{t t}\right\rangle_{\text {vacuum }}+\left\langle T_{t t}\right\rangle_{\text {mixed }}+\left\langle T_{t t}\right\rangle_{\text {thermal }},
$$

where the first and last terms are the (two-dimensional versions of the) well-known expressions for the "Casimir" energy density and the "blackbody" energy density, respectively,

$$
\left\langle T_{t t}\right\rangle_{\text {vacuum }}=-\frac{\pi}{6 a^{2}}, \quad\left\langle T_{t t}\right\rangle_{\text {thermal }}=\frac{\pi}{6} T^{2} .
$$

The second term in Eq. (22) arises when the differential operators in Eq. (9) act on $G_{\text {mixed }}\left(\mathrm{x}, \mathrm{x}^{\prime}\right)$ in Eq. (16), and therefore it contains a double series. When $N=4$ (which was the case in Refs. $[13,16])$, the order in which the corresponding summations are evaluated is irrelevant since the double series is absolutely convergent [17]. However, when $N=2$ the double series is not absolutely convergent and the order of evaluation of the summations does matter, as will now be shown.

One may first sum over the "boundary" number $n$, corresponding to

$$
\begin{aligned}
\left\langle T_{t t}\right\rangle_{\text {mixed }} & =T^{2} u(T a), \\
u(q) & :=\frac{2}{\pi} \sum_{m=1}^{\infty} \sum_{n=1}^{\infty} \frac{m^{2}-q^{2} n^{2}}{\left(m^{2}+q^{2} n^{2}\right)^{2}} .
\end{aligned}
$$

Or, instead, one may first sum over the "thermal" number $m$, i.e.,

$$
\begin{aligned}
\left\langle T_{t t}\right\rangle_{\text {mixed }} & =T^{2} v(T a), \\
v(q) & :=\frac{2}{\pi} \sum_{n=1}^{\infty} \sum_{m=1}^{\infty} \frac{m^{2}-q^{2} n^{2}}{\left(m^{2}+q^{2} n^{2}\right)^{2}} .
\end{aligned}
$$

It can be noticed that

$$
v(q)=-\frac{1}{q^{2}} u(1 / q) .
$$

In fact, using Ref. [26] or Ref. [27], the first summations in Eqs. (24) and (25) can be evaluated to give

$$
\begin{aligned}
& u(q)=-\frac{\pi}{6}+\frac{\pi}{q^{2}} \sum_{k=1}^{\infty} \operatorname{cosech}^{2}\left(\frac{k \pi}{q}\right), \\
& v(q)=\frac{\pi}{6 q^{2}}-\pi \sum_{k=1}^{\infty} \operatorname{cosech}^{2}(k \pi q) .
\end{aligned}
$$

These expressions can be compared with each other by using the identity

$$
\sum_{k=1}^{\infty} \frac{k}{e^{2 \pi q k}-1}=\frac{1}{4} \sum_{k=1}^{\infty} \operatorname{cosech}^{2}(k \pi q),
$$


to recast "Schlömilch's formula" as ${ }^{3}$

$$
\begin{aligned}
- & \pi q \sum_{k=1}^{\infty} \operatorname{cosech}^{2}(k \pi q)-\frac{\pi}{q} \sum_{k=1}^{\infty} \operatorname{cosech}^{2}\left(\frac{k \pi}{q}\right) \\
& =1-\frac{\pi}{6}\left(q+\frac{1}{q}\right),
\end{aligned}
$$

where one can appreciate the symmetry $q \rightarrow 1 / q$. Considering then Eq. (29) in Eq. (27), it follows that

$$
v(q)=u(q)+\frac{1}{q},
$$

showing that the double series in Eqs. (24) and (25) are indeed distinct. Now, noting Eqs. (22), (23), and (27), $u(T a)$ in Eq. (24) leads to

$$
\left\langle T_{t t}\right\rangle=-\frac{\pi}{6 a^{2}}+\frac{\pi}{a^{2}} \sum_{k=1}^{\infty} \operatorname{cosech}^{2}\left(\frac{k \pi}{T a}\right),
$$

whereas $v(T a)$ in Eq. (25) leads to

$$
\left\langle T_{t t}\right\rangle=-\frac{\pi}{6 a^{2}}+\frac{T}{a}+\frac{\pi}{a^{2}} \sum_{k=1}^{\infty} \operatorname{cosech}^{2}\left(\frac{k \pi}{T a}\right),
$$

where Eq. (30) has been used. Thus, one ends up with two expressions for the energy density that differ from each other by a term linear in temperature: Eq. (31) (which is obtained by "summing first over $n$ ") and Eq. (32) (which arises by "summing first over $m$ "). In fact, by taking into account Eq. (21), it is seen that for each order of summation chosen it corresponds to a different $\left\langle T_{\mu \nu}\right\rangle$. It should be noticed that Eqs. (21) and (31) are the result reported in the literature [4] (i.e., "summing first over $n$ ").

Noting Eqs. (21), (31), and (32), one sees that the terms carrying $\xi$ in Eqs. (9)-(12) all cancel each other, and that $\left\langle T_{\mu \nu}\right\rangle$ is traceless, as it should be. Also, since $\left\langle T_{\mu \nu}\right\rangle$ is stationary and homogeneous, it is trivially conserved, i.e., $\left\langle T^{\mu \nu}\right\rangle_{, \nu}=0$.

The physics of the ambiguity in Eqs. (31) and (32), together with Eq. (21), can be better explored by considering the asymptotic behaviors of $\left\langle T_{\mu \nu}\right\rangle$. This is done next.

\section{Summing first over $n$}

Using Eq. (28) in the expression for $u$ in Eq. (27), some manipulation leads to

\footnotetext{
"'Schlömilch's formula" is a Ramanujan-type identity which has been used throughout the literature in related contexts. See, e.g., Eq. (40) in Ref. [28] and Eq. (1) in Ref. [29]. [A typo was detected in Eq. (40) of Ref. [28]: the term $1 / 2 \pi$ should be replaced by $1 / 2$.]
}

$$
u(q \rightarrow 0)=-\frac{\pi}{6}+\frac{4 \pi}{q^{2}} e^{-2 \pi / q},
$$

where smaller exponential corrections have been omitted (and will be omitted throughout the text). Then, at low temperatures or for small circles, Eqs. (22) to (24) with Eq. (33) yield

$$
\left\langle T_{t t}\right\rangle=-\frac{\pi}{6 a^{2}}+\frac{4 \pi}{a^{2}} e^{-2 \pi / T a}, \quad T a \ll 1,
$$

showing that the correction to the "Casimir" energy density [see Eq. (23)] decreases exponentially when $T a \rightarrow 0$.

Considering now Eqs. (26) and (30), we obtain

$$
u(q)=-\frac{1}{q}-\frac{1}{q^{2}} u(1 / q)
$$

which with Eq. (33) gives

$$
u(q \rightarrow \infty)=-\frac{1}{q}+\frac{\pi}{6 q^{2}}-4 \pi e^{-2 \pi q} .
$$

Using now Eq. (36) in Eq. (24), one obtains from Eqs. (22) and (23) that

$$
\left\langle T_{t t}\right\rangle=\frac{\pi}{6} T^{2}-\frac{T}{a}-4 \pi T^{2} e^{-2 \pi T a}, \quad T a \gg 1 .
$$

Thus, the "blackbody" energy density [see Eq. (23)] drops by $T / a$ at high temperatures or for big circles.

It should be remarked that the behavior of $u$ for large values of $q$ [see Eq. (36)] has been determined from its behavior for small values of $q$ [see Eq. (33)] through Eq. (35). Such a feature is typical of quantum fields at finite temperature in backgrounds with boundaries and has been well known in the literature for a long time [13,30]. It is also worth remarking that the "blackbody"-like energy density in Eq. (37) and the "Casimir"-like energy density in Eq. (34) correspond to different regimes (of temperature and size) of the very same phenomenon.

\section{Summing first over $\boldsymbol{m}$}

Noting Eqs. (31) and (32) and the text just after them, one sees that the easiest way to get the asymptotic behaviors corresponding to "summing first over $m$ " is to add $T / a$ to Eqs. (34) and (37), namely,

$$
\left\langle T_{t t}\right\rangle=-\frac{\pi}{6 a^{2}}+\frac{T}{a}+\frac{4 \pi}{a^{2}} e^{-2 \pi / T a}, \quad T a \ll 1
$$

and

$$
\left\langle T_{t t}\right\rangle=\frac{\pi}{6} T^{2}-4 \pi T^{2} e^{-2 \pi T a}, \quad T a \gg 1 .
$$


Alternatively, one could proceed as above, using Eq. (28) in the expression for $v$ in Eq. (27), to obtain

$$
v(q \rightarrow \infty)=\frac{\pi}{6 q^{2}}-4 \pi e^{-2 \pi q} .
$$

Then, Eqs. (22), (23), and (25) with Eq. (40) yield Eq. (39). Now, from Eqs. (26) and (30), it follows that

$$
v(q)=\frac{1}{q}-\frac{1}{q^{2}} v(1 / q),
$$

which, combined with Eq. (40), gives

$$
v(q \rightarrow 0)=\frac{1}{q}-\frac{\pi}{6}+\frac{4 \pi}{q^{2}} e^{-2 \pi / q} .
$$

Considering again Eqs. (22), (23), and (25) now with Eq. (42), one ends up with Eq. (38).

In comparing Eq. (34) with Eq. (38) and Eq. (37) with Eq. (39), we see that the small exponential correction to the "Casimir" energy density has been replaced by a linear one, and that the linear correction to the "blackbody" energy density has been replaced by a small exponential correction. These modifications will have radical consequences in thermodynamics, as will be shown shortly.

\section{B. Local thermodynamic stability}

It is natural to wonder whether the requirement of local thermodynamic stability (see, e.g., Sec. IV in Ref. [31]) might resolve the ambiguity in Eqs. (21), (31), and (32). Consider a small segment of the circle, and assume that the temperature $T_{\text {in }}$ inside the segment differs (due to a fluctuation) slightly from $T_{\text {out }}$, which is the temperature outside the segment. Conservation of momentum dictates that the power (energy per unity of time) radiated out of the segment is proportional to the differences of stresses inside and outside, i.e.,

$$
\Phi=\left\langle T_{x x}\right\rangle_{\text {in }}-\left\langle T_{x x}\right\rangle_{\text {out }},
$$

up to a positive overall factor [31]. Below, the regimes $T a \ll 1$ and $T a \gg 1$ will be investigated.

\section{Summing first over $n$}

Using Eqs. (21) and (34) in Eq. (43) gives

$$
\Phi=\frac{4 \pi}{a^{2}}\left(e^{-2 \pi / a T_{\text {in }}}-e^{-2 \pi / a T_{\text {out }}}\right), \quad T a \ll 1 .
$$

Say that $T_{\text {in }}>T_{\text {out }}$, i.e., $\Phi$ in Eq. (44) is positive. Taking the derivative with respect to temperature of the energy density in Eq. (34), it follows that

$$
\frac{\partial}{\partial T}\left\langle T_{t t}\right\rangle=\frac{8 \pi^{2}}{T^{2} a^{3}} e^{-2 \pi / T a}>0 .
$$

Thus, as $\Phi>0$, energy will leave the segment. Due to conservation of energy (i.e., energy in the segment will decrease) and noticing Eq. (45), $T_{\text {in }}$ will drop with the thermodynamic equilibrium being restored, as expected.

Considering now Eqs. (21) and (37) in Eq. (43), the leading contribution is

$$
\Phi=\frac{\pi}{6}\left(T_{\text {in }}^{2}-T_{\text {out }}^{2}\right), \quad T a \gg 1 .
$$

If $T_{\text {in }}>T_{\text {out }}, \Phi$ in Eq. (46) is positive and energy will leave the segment. As $\partial_{T}\left\langle T_{t t}\right\rangle>0$ [from Eq. (37)], conservation of energy determines that $T_{\text {in }}$ will drop, and thermodynamic equilibrium will be restored again.

These results show that "summing first over $n$ " is consistent with local thermodynamic stability.

\section{Summing first over $\boldsymbol{m}$}

Considering Eqs. (21) and (38), Eq. (43) yields

$$
\Phi=\frac{1}{a}\left(T_{\text {in }}-T_{\text {out }}\right), \quad T a \ll 1,
$$

up to small exponential corrections. Taking into account the leading contribution in Eq. (38),

$$
\frac{\partial}{\partial T}\left\langle T_{t t}\right\rangle=\frac{1}{a}>0 .
$$

By repeating the argument above, if $T_{\text {in }}>T_{\text {out }}$ in Eq. (47), then $\Phi>0$ and energy leaves the segment. It follows then from Eq. (48) that $T_{\text {in }}$ drops, i.e., thermodynamic equilibrium is recovered.

By using Eqs. (21) and (39) in Eq. (43), one also ends up with Eq. (46). The same argument just after Eq. (46) shows that here as well thermodynamic equilibrium will be restored.

Therefore, "summing first over $m$ " is also consistent with local thermodynamic stability.

\section{Thermodynamics}

The first step to obtain thermodynamics in the "local approach" is to integrate the energy density over the box, i.e.,

$$
U=\int_{0}^{a}\left\langle T_{t t}\right\rangle d x,
$$

yielding the internal energy $U$. By noticing the identity in Eq. (28) and considering Eq. (49), the homogeneous $\left\langle T_{t t}\right\rangle$ 's in Eqs. (31) ("summing first over $n$ ") and (32) ("summing first over $m$ ") lead to the contrasting expressions for $U$ in Eqs. (2) and (4), respectively, which as mentioned 
previously are the source of the zero-mode controversy in the "global approach" [see the text just after Eq. (4)]. The thermodynamic aspects of this ambiguity are also better appreciated by looking at the asymptotic behaviors.

\section{Summing first over $n$}

Corresponding to Eq. (34) one has from Eq. (49) that

$$
U(T, a)=-\frac{\pi}{6 a}+\frac{4 \pi}{a} e^{-2 \pi / T a}, \quad T a \ll 1,
$$

in agreement with an early calculation using the partition function (i.e., the "global approach") on the circle [18]. As the heat capacity at constant volume is positive, i.e., $C:=\partial_{T} U>0$ [see Eq. (45)], one of the criteria for global thermodynamic stability is satisfied [22].

Now, using Eq. (50) in Eq. (5) and integrating, we obtain

$$
F(T, a)=-\frac{\pi}{6 a}-2 T e^{-2 \pi / T a}, \quad T a \ll 1,
$$

where the integration constant has been set such that the thermodynamic pressure $\mathrm{p}:=-\partial_{a} F$ equals the stress $\left\langle T_{x x}\right\rangle$ [see Eqs. (21) and (34)]. It should be noticed that, since $\left\langle T_{\mu \nu}\right\rangle$ is traceless, the equation of state

$$
U=\mathrm{p} a
$$

holds, and that the "Casimir force" $-\pi / 6 a^{2}$ (which tends to contract the circle) is weakened by an small exponential "thermal" contribution [see p in Eq. (34)]. The entropy $S=-\partial_{T} F$ following from Eq. (51) is given by

$$
S(T, a)=2\left(\frac{2 \pi}{T a}+1\right) e^{-2 \pi / T a}, \quad T a \ll 1 .
$$

Then, when $T \rightarrow 0, S$ in Eq. (53) vanishes, i.e., the third law of thermodynamics is satisfied.

Now, corresponding to the energy density in Eq. (37) one has that

$$
U(T, a)=\frac{\pi}{6} a T^{2}-T-4 \pi a T^{2} e^{-2 \pi T a}, \quad T a \gg 1,
$$

which agrees with early calculations where the "global approach" was used $[18,19]$. It follows from Eq. (54) that $C:=\partial_{T} U>0$, which as mentioned previously is one of the criteria for global thermodynamic stability [22]. Following the same steps applied in the regime $T a \ll 1$ above, it results that [see Eq. (5)]

$$
F(T, a)=-\frac{\pi}{6} a T^{2}+T \ln (T a)-2 T e^{-2 \pi T a}, \quad T a \gg 1,
$$

where Eq. (52) still holds, i.e., p is given by Eq. (37) where $\pi T^{2} / 6$ is the "blackbody radiation force" and $-T / a$ is the "thermal Casimir force" (which, unlike the blackbody contribution, tends to contract the circle). The asymptotic behavior of the entropy associated with Eq. (55) is

$$
\begin{gathered}
S(T, a)=\frac{\pi}{3} a T-[\ln (T a)+1]-2(2 \pi T a-1) e^{-2 \pi T a}, \\
T a \gg 1,
\end{gathered}
$$

which becomes the entropy of the "blackbody radiation," $\pi a T / 3$, as $T a \rightarrow \infty$.

In both regimes above (i.e., $T a \ll 1$ and $T a \gg 1$ ) one can check that $\partial_{a} p>0$, which, in fact, violates one of the criteria for global thermodynamic stability ${ }^{4}$ [22].

\section{Summing first over $m$}

Repeating the procedures above, the $U$ corresponding to Eq. (38) is given by Eq. (50) after adding $T$, from which it follows that

$F(T, a)=-\frac{\pi}{6 a}-T \ln (T a)-2 T e^{-2 \pi / T a}, \quad T a \ll 1$,

instead of Eq. (51). The equation of state (52) holds, and therefore the "Casimir force" is now weakened by a term linear in temperature [see $p$ in Eq. (38)]. It follows from Eq. (57) that

$S(T, a)=\ln (T a)+1+2\left(\frac{2 \pi}{T a}+1\right) e^{-2 \pi / T a}, \quad T a \ll 1$,

which clearly violates the third law of thermodynamics, with the entropy $S$ diverging to $-\infty$ as $T \rightarrow 0$. This fact is sometimes used in the literature to argue that the zero mode should not be taken into account in computations of the partition function (see e.g., Refs. [7,9]), i.e., one should "sum first over $n$ " accordingly.

Turning now to the regime $T a \gg 1$, corresponding to Eq. (39), it follows that $U$ is given in Eq. (54) by omitting $-T, F$ is given in Eq. (55) by omitting $T \ln (T a)$, and $S$ is given in Eq. (56) by omitting $-\ln (T a)-1$. The equation of state (52) holds, and p in Eq. (39) shows that this time there is no "thermal Casimir force".

In both regimes it can be checked that again $C>0$ and $\partial_{a} \mathrm{p}>0$.

\section{INTERVAL WITH REFLECTING EDGES}

In this section we address the thermal behavior of a massless scalar field in an interval where Dirichlet or Neumann boundary conditions are taken at the end points, $x=0$ and $x=a$. That is, the end points are the reflecting "walls" of a one-dimensional box containing hot scalar radiation in flat two-dimensional spacetime [see Eq. (1)].

\footnotetext{
${ }^{4} C>0$ implies thermal stability; $\partial_{a} \mathrm{p}<0$ implies mechanical stability.
} 


\section{A. $\left\langle\boldsymbol{T}_{\boldsymbol{\mu}}\right\rangle$}

In order to determine $\left\langle T_{\mu \nu}\right\rangle$, we again use Eqs. (8)-(12), but now with the Feynman propagator given by [see Eqs. (A16)-(A17) and the end of Appendix A 2]

$$
G_{\mathcal{F}}\left(\mathrm{x}, \mathrm{x}^{\prime}\right)=\sum_{m=-\infty}^{\infty} \sum_{n=-\infty}^{\infty}\left[f\left(\sigma_{-}^{(m, n)}\right) \mp f\left(\sigma_{+}^{(m, n)}\right)\right]
$$

with $f$ is defined in Eq. (14) and we recall that the upper and lower signs are for Dirichlet and Neumann boundary conditions, respectively. Removing the Minkowski vacuum propagator $G_{0}\left(\mathrm{x}, \mathrm{x}^{\prime}\right)$ from $G_{\mathcal{F}}\left(\mathrm{x}, \mathrm{x}^{\prime}\right)$ in Eq. (15) [note that $\sigma^{(m, 0)}=\sigma_{-}^{(m, 0)}$, we obtain the following renormalized propagator:

$$
\begin{aligned}
G\left(\mathrm{x}, \mathrm{x}^{\prime}\right)= & G_{\text {vacuum }}^{\text {Casimir }}\left(\mathrm{x}, \mathrm{x}^{\prime}\right)+G_{\text {vacuum }}^{\text {wall }}\left(\mathrm{x}, \mathrm{x}^{\prime}\right)+G_{\text {mixed }}^{\text {Casimir }}\left(\mathrm{x}, \mathrm{x}^{\prime}\right) \\
& +G_{\text {mixed }}^{\text {wall }}\left(\mathrm{x}, \mathrm{x}^{\prime}\right)+G_{\text {thermal }}\left(\mathrm{x}, \mathrm{x}^{\prime}\right),
\end{aligned}
$$

where $G_{\text {thermal }}\left(\mathrm{x}, \mathrm{x}^{\prime}\right)$ is the "blackbody" propagator in Eq. (16), and

$$
\begin{gathered}
G_{\text {vacuum }}^{\text {Casimir }}\left(\mathrm{x}, \mathrm{x}^{\prime}\right):=\sum_{n=-\infty}^{\infty} f\left(\sigma_{-}^{(0, n)}\right), \\
G_{\text {vacuum }}^{\text {wall }}\left(\mathrm{x}, \mathrm{x}^{\prime}\right):=\mp \sum_{n=-\infty}^{\infty} f\left(\sigma_{+}^{(0, n)}\right), \\
G_{\text {mixed }}^{\text {Casimir }}\left(\mathrm{x}, \mathrm{x}^{\prime}\right):=\sum_{m=-\infty}^{\infty} \sum_{n=-\infty}^{\infty} f\left(\sigma_{-}^{(m, n)}\right), \\
G_{\text {mixed }}^{\text {wall }}\left(\mathrm{x}, \mathrm{x}^{\prime}\right):=\mp \sum_{m=-\infty}^{\infty} \sum_{n=-\infty}^{\infty} f\left(\sigma_{+}^{(m, n)}\right) .
\end{gathered}
$$

One sees from these definitions and Eq. (14) that, when $T \rightarrow 0$, only the vacuum contributions remain in Eq. (60). When $a \rightarrow \infty$, both $G_{\text {vacuum }}^{\text {Casimir }}\left(\mathrm{x}, \mathrm{x}^{\prime}\right)$ and $G_{\text {mixed }}^{\text {Casimir }}\left(\mathrm{x}, \mathrm{x}^{\prime}\right)$ vanish. Thus, when $T \rightarrow 0$ and $a \rightarrow \infty$, only $G_{\text {vacuum }}^{\text {wall }}\left(\mathrm{x}, \mathrm{x}^{\prime}\right)$ is left in Eq. (60).

Proceeding now as in Sec. II A and considering $N=2$ and $M \rightarrow 0$, Eqs. (60) and (19) are used in Eqs. (9)-(12) to obtain the four components of $\left\langle T_{\mu \nu}\right\rangle$ :

$$
\begin{aligned}
\left\langle T_{t t}\right\rangle= & \left\langle T_{t t}\right\rangle_{\text {vacuum }}^{\text {Casimir }}+\left\langle T_{t t}\right\rangle_{\text {vacuum }}^{\text {wall }}+\left\langle T_{t t}\right\rangle_{\text {mixed }}^{\text {Casimir }} \\
& +\left\langle T_{t t}\right\rangle_{\text {mixed }}^{\text {wall }}+\left\langle T_{t t}\right\rangle_{\text {thermal }}
\end{aligned}
$$

and,

\footnotetext{
${ }^{5}$ When $a \rightarrow \infty$ is set in the $\left\langle T_{\mu \nu}\right\rangle$ obtained in this section, formulas corresponding to the presence of a single reflecting wall at $x=0$ are consistently reproduced (see Refs. [20,24,32]).
}

$$
\begin{aligned}
& \left\langle T_{x x}\right\rangle=\left\langle T_{t t}\right\rangle_{\text {vacuum }}^{\text {Casimir }}+\left\langle T_{t t}\right\rangle_{\text {mixed }}^{\text {Casimir }}+\left\langle T_{t t}\right\rangle_{\text {thermal }}, \\
& \left\langle T_{t x}\right\rangle=\left\langle T_{x t}\right\rangle=0 .
\end{aligned}
$$

The contribution $\left\langle T_{t t}\right\rangle_{\text {thermal }}$ is the "blackbody" energy density in Eq. (23), and

$$
\begin{aligned}
\left\langle T_{t t}\right\rangle_{\text {vacuum }}^{\text {Casimir }} & =-\frac{\pi}{24 a^{2}}, \\
\left\langle T_{t t}\right\rangle_{\text {vacuum }}^{\text {wall }} & = \pm \xi \frac{\pi}{2 a^{2}} \csc ^{2}\left(\frac{\pi x}{a}\right)
\end{aligned}
$$

are the vacuum energy densities. The contributions $\left\langle T_{t t}\right\rangle_{\text {mixed }}^{\text {Casimir }}$ and $\left\langle T_{t t}\right\rangle_{\text {mixed }}^{\text {wall }}$, which contain double series [see Eq. (62)] and are the source of ambiguities, will be treated shortly.

As already mentioned, we have not found any study of $\left\langle T_{\mu \nu}\right\rangle$ in the literature for hot scalar radiation in an interval with reflecting edges. However, there have been studies of $\left\langle T_{\mu \nu}\right\rangle$ at zero temperature [21,33], and the sum of the contributions in Eq. (65) is in agreement with the vacuum energy density calculated in those references.

The ambiguity in the value of the homogeneous $\left\langle T_{t t}\right\rangle_{\text {mixed }}^{\text {Casimir }}$ corresponds to that in Eqs. (24) and (25), namely,

$$
\left\langle T_{t t}\right\rangle_{\text {mixed }}^{\text {Casimir }}=T^{2} u(2 T a), \quad\left\langle T_{t t}\right\rangle_{\text {mixed }}^{\text {Casimir }}=T^{2} v(2 T a),
$$

where Eq. (30) should be noticed. The ambiguity in the value of the nonhomogeneous $\left\langle T_{t t}\right\rangle_{\text {mixed }}^{\text {wall }}$ is new. Again, one may sum first over the "boundary" number $n$, i.e.,

$$
\begin{aligned}
\left\langle T_{t t}\right\rangle_{\text {mixed }}^{\text {wall }} & =\mp 2 \xi T^{2} \mu(2 x / a, T a), \\
\mu(p, q) & :=\frac{2}{\pi} \sum_{m=1}^{\infty} \sum_{n=-\infty}^{\infty} \frac{m^{2}-q^{2}(p-2 n)^{2}}{\left[m^{2}+q^{2}(p-2 n)^{2}\right]^{2}} .
\end{aligned}
$$

But one may also sum first over the "thermal" number $m$, i.e.,

$$
\begin{aligned}
\left\langle T_{t t}\right\rangle_{\text {mixed }}^{\text {wall }} & =\mp 2 \xi T^{2} \nu(2 x / a, T a), \\
\nu(p, q) & :=\frac{2}{\pi} \sum_{n=-\infty}^{\infty} \sum_{m=1}^{\infty} \frac{m^{2}-q^{2}(p-2 n)^{2}}{\left[m^{2}+q^{2}(p-2 n)^{2}\right]^{2}} .
\end{aligned}
$$

An interesting fact to point out is that for $\xi=0$ (i.e., for minimal and conformal couplings) the "wall" ambiguity in Eqs. (67) and (68) disappears, whereas the "Casimir" ambiguity in Eq. (66) remains. In fact, when $\xi=0$, the expressions for the components of $\left\langle T_{\mu \nu}\right\rangle$ in an interval with reflecting edges are given by those for the circle in Eqs. (21), (31), and (32), after replacing $a$ by $2 a$ [see Eqs. (63) and (64)]. 
Note that the symmetry

$\mu(2-p, q)=\mu(p, q), \quad \nu(2-p, q)=\nu(p, q)$

was already expected since the two identical reflecting walls are sitting at $x=0$ and at $x=a$. By setting $p=2 x / a, x=0$ and $x=a$ correspond to $p=0$ and $p=2$, respectively. Regarding $\left\langle T_{t t}\right\rangle_{\text {mixed }}^{\text {wall in Eqs. (67) }}$ and (68), it follows that one can consider $p$ running from 0 to 1 , using then Eq. (69) to determine $\left\langle T_{t t}\right\rangle_{\text {mixed }}^{\text {wall }}$ in the other half of the interval, i.e., for $x>a / 2$. [Clearly the same remark applies to $\left\langle T_{t t}\right\rangle_{\text {vacuum }}^{\text {wall }}$ in Eq. (65).]

A comparison of $\mu$ and $\nu$ in Eqs. (67) and (68) with $u$ and $v$ in Eqs. (24) and (25) shows that (below, $2 q$ is the argument of the functions $u$ and $v$ )

$\mu(0, q)=2 u(2 q)+\frac{\pi}{3}, \quad \nu(0, q)=2 v(2 q)+\frac{\pi}{3}$.

Using now Eq. (30), Eq. (70) yields

$$
\nu(0, q)=\mu(0, q)+\frac{1}{q} .
$$

Then, taking into account Eq. (69), it follows that the relation in Eq. (71) also holds when $p=2$. Indeed, these facts suggest that the relation in Eq. (71) may hold for arbitrary $p \in[0,2]$, i.e.:

Conjecture:

$$
\nu(p, q)=\mu(p, q)+\frac{1}{q} .
$$

We do not have a proof of the equality in Eq. (72) for arbitrary $p$, although there is strong numerical evidence that supports it [27]. The main reason to display the conjecture as in Eq. (72) is to check its consistency with results that will appear later in the text. ${ }^{6}$

The summation over $n$ in Eq. (67) can be evaluated (by using, e.g., Ref. [27]) to give

$$
\begin{aligned}
\mu(p, q)= & -\frac{\pi}{4 q^{2}} \sum_{k=1}^{\infty}\left[\csc ^{2}\left\{\frac{\pi(p q-i k)}{2 q}\right\}\right. \\
& \left.+\csc ^{2}\left\{\frac{\pi(p q+i k)}{2 q}\right\}\right] .
\end{aligned}
$$

By using trigonometric and hyperbolic identities and after some manipulations, Eq. (73) can be recast as

\footnotetext{
${ }^{6}$ It should be stressed that there is a proof of Eq. (72) when $p=0$ and $p=2$, as has been shown. The proof when $p$ is arbitrary possibly involves some generalization of "Schlömilch's formula," which may turn out to be a hard task.
}

$$
\mu(p, q)=\frac{\pi}{q^{2}} \sum_{k=1}^{\infty} \frac{\cos (p \pi) \cosh (k \pi / q)-1}{[\cos (p \pi)-\cosh (k \pi / q)]^{2}} .
$$

The first summation in Eq. (68) can also be evaluated [27] to give

$$
\begin{aligned}
\nu(p, q)= & \frac{\pi}{4 q^{2}} \csc ^{2}\left(\frac{p \pi}{2}\right)-\pi \operatorname{cosech}^{2}(p q \pi) \\
& -\pi \sum_{k=1}^{\infty}\left[\operatorname{cosech}^{2}\{q \pi(2 k-p)\}\right. \\
& \left.+\operatorname{cosech}^{2}\{q \pi(2 k+p)\}\right],
\end{aligned}
$$

where it should be noticed that although each of the first two terms diverges as $p \rightarrow 0$, their sum remains finite, i.e.,

$\lim _{p \rightarrow 0}\left[\frac{\pi}{4 q^{2}} \csc ^{2}\left(\frac{p \pi}{2}\right)-\pi \operatorname{cosech}^{2}(p q \pi)\right]=\frac{\pi}{12 q^{2}}+\frac{\pi}{3}$.

Taking into account the dependence on $\xi$ in Eqs. (65), (67), and (68), one sees that the stationary $\left\langle T_{\mu \nu}\right\rangle$ in Eqs. (63) and (64) is traceless when $\xi=0$, and that since the stress $\left\langle T_{x x}\right\rangle$ is homogeneous, $\left\langle T^{\mu \nu}\right\rangle_{, \nu}=0$.

As for the case of the circle in the previous section, the physics of $\left\langle T_{\mu \nu}\right\rangle$ in Eqs. (63) and (64) can be better studied by looking at the thermal behaviors of $\left\langle T_{\mu \nu}\right\rangle$ corresponding to $T a \ll 1$ and $T a \gg 1$. However, it is worth remarking that now $\left\langle T_{\mu \nu}\right\rangle$ is nonhomogeneous, i.e., its value near one of the walls (say, $x \approx 0$ ) is different from that in the bulk of the box (say, $x \approx a / 2$ ). Before embarking on this study, one has to decide which summations are going to be considered first in the expressions for $\left\langle T_{t t}\right\rangle_{\text {mixed }}^{\text {Casimir }}$ and $\left\langle T_{t t}\right\rangle_{\text {mixed }}^{\text {wall }}$ [see Eqs. (66), (67), and (68)]. For the sake of consistency, the same order of summation will be taken in both expressions. Note that the expression for $\left\langle T_{x x}\right\rangle$ can be obtained from that for $\left\langle T_{t t}\right\rangle$ simply by setting $\xi=0$ in the latter [see Eq. (64)], i.e.,

$$
\left\langle T_{x x}\right\rangle=\left\langle T_{t t}\right\rangle_{\xi=0},
$$

showing explicitly the independence of the stress of $\xi$ and thus of the type of boundary condition.

\section{Summing first over $n$}

Looking at Eq. (74), it quickly follows that

$$
\mu(p, q \rightarrow 0)=\frac{2 \pi}{q^{2}} \cos (p \pi) e^{-\pi / q},
$$

where (as already mentioned) smaller exponential terms are being omitted. Now, using Eq. (33) in Eq. (66) and Eq. (78) in Eq. (67), and at the same time noting Eqs. (23) and (65), Eq. (63) gives 


$$
\begin{aligned}
\left\langle T_{t t}\right\rangle= & -\frac{\pi}{24 a^{2}} \pm \xi \frac{\pi}{2 a^{2}} \csc ^{2}\left(\frac{\pi x}{a}\right) \\
& +\left[1 \mp 4 \xi \cos \left(\frac{2 \pi x}{a}\right)\right] \frac{\pi}{a^{2}} e^{-\pi / T a}, \quad T a \ll 1,
\end{aligned}
$$

which holds not only for low temperatures, but also for arbitrary temperatures and small enough boxes. It should be noted that, although the correction in Eq. (79) to the vacuum energy density is exponentially small, it will turn out to be relevant when certain thermodynamic issues are addressed later in the text.

Regarding the nonhomogeneous energy density in Eq. (79), two places in the box are of particular interest: very close to one wall (say, the wall at $x=0$ ),

$$
\begin{aligned}
\left\langle T_{t t}\right\rangle= & \pm \frac{\xi}{2 \pi x^{2}}+(1 \mp 4 \xi)\left[-\frac{\pi}{24 a^{2}}+\frac{\pi}{a^{2}} e^{-\pi / T a}\right], \\
T a & \ll 1, \quad x / a \ll 1,
\end{aligned}
$$

and at the middle of the box,

$$
\begin{gathered}
\left\langle T_{t t}\right\rangle=-\frac{\pi}{24 a^{2}} \pm \xi \frac{\pi}{2 a^{2}}+(1 \pm 4 \xi) \frac{\pi}{a^{2}} e^{-\pi / T a}, \\
T a \ll 1, \quad x=a / 2 .
\end{gathered}
$$

When $\xi \neq 0$, the first term in Eq. (80) carries a nonintegrable divergence, corresponding to $x \rightarrow 0$, which is well known in the literature of vacuum fluctuations in boxes with reflecting walls [21]. In the bulk of the box, it is seen from Eq. (81) that the "Casimir" vacuum energy density can be substantially modified by a nonvanishing $\xi$.

In order to obtain the behavior corresponding to $T a \gg 1$, one can proceed essentially along the same steps that led from Eq. (63) to Eq. (79). But now it should be noticed that, keeping $0<p \lesssim 1$, Eq. (75) yields

$$
\begin{aligned}
\nu(p, q \rightarrow \infty)= & \frac{\pi}{4 q^{2}} \csc ^{2}\left(\frac{p \pi}{2}\right)-\pi \operatorname{cosech}^{2}(p q \pi) \\
& -8 \pi e^{-4 \pi q} \cosh (2 p q \pi) .
\end{aligned}
$$

Then, using Eq. (36) in Eqs. (66) and (82) in Eq. (72), it results that

$$
\begin{aligned}
\left\langle T_{t t}\right\rangle= & \pm 2 \pi \xi T^{2} \operatorname{cosech}^{2}(2 \pi T x)+\frac{\pi}{6} T^{2}-(1 \mp 4 \xi) \frac{T}{2 a} \\
& -4 \pi T^{2}[1 \mp 4 \xi \cosh (4 \pi T x)] e^{-4 \pi T a}, \quad T a \gg 1
\end{aligned}
$$

for $0<x \lesssim a / 2$. As it stands, Eq. (83) is also a conjecture, except when $x / a \rightarrow 0$, in which case Eq. (71) can be used, i.e.,

$$
\begin{aligned}
&\left\langle T_{t t}\right\rangle= \pm \frac{\xi}{2 \pi x^{2}}+(1 \mp 4 \xi)\left[\frac{\pi}{6} T^{2}-\frac{T}{2 a}-4 \pi T^{2} e^{-4 \pi T a}\right], \\
& T a \gg 1, \quad x / a \ll 1 .
\end{aligned}
$$

It is worth noting that the "Casimir" vacuum energy density in Eq. (80) (first term between right brackets) and the "blackbody" energy density in Eq. (84) play similar roles, with the latter diminished by a linear term in $T / a$.

The behavior of $\left\langle T_{t t}\right\rangle$ in the bulk, for high temperatures or large boxes [which should be confronted with that in Eq. (81)], can be obtained from Eq. (83),

$$
\begin{gathered}
\left\langle T_{t t}\right\rangle=\frac{\pi}{6} T^{2}-(1 \mp 4 \xi) \frac{T}{2 a} \pm 16 \pi \xi T^{2} e^{-2 \pi T a}, \\
T a \gg 1, \quad x=a / 2,
\end{gathered}
$$

which is essentially the "blackbody" energy density, but corrected by a term linear in $T / a$ that depends on $\xi$. [Recall that Eq. (85), though numerically supported [27], is a conjecture.]

As has been previously mentioned, expressions for the stress $\left\langle T_{x x}\right\rangle$ can be obtained from those for $\left\langle T_{t t}\right\rangle$ above, as prescribed in Eq. (77). An example is perhaps instructive. For instance, Eq. (81) corresponds to

$$
\left\langle T_{x x}\right\rangle=-\frac{\pi}{24 a^{2}}+\frac{\pi}{a^{2}} e^{-\pi / T a}, \quad T a \ll 1, \quad x=a / 2,
$$

which is essentially the familiar "Casimir" effect: a vacuum force attracting two reflecting walls.

\section{Summing first over $m$}

Starting with Eq. (63) and assuming Eq. (72), it is straightforward to show that $\left\langle T_{t t}\right\rangle$ corresponding to "summing first over $m$ " should be obtained from that for "summing first over $n$ " by adding the following homogeneous term:

$$
(1 \mp 4 \xi) \frac{T}{2 a} \text {. }
$$

According to this prescription, e.g., Eqs. (80) and (81) lead to

$$
\begin{aligned}
&\left\langle T_{t t}\right\rangle= \pm \frac{\xi}{2 \pi x^{2}}+(1 \mp 4 \xi)\left[-\frac{\pi}{24 a^{2}}+\frac{T}{2 a}+\frac{\pi}{a^{2}} e^{-\pi / T a}\right], \\
& T a \ll 1, \quad x / a \ll 1,
\end{aligned}
$$

and

$$
\begin{gathered}
\left\langle T_{t t}\right\rangle=-\frac{\pi}{24 a^{2}} \pm \xi \frac{\pi}{2 a^{2}}+(1 \mp 4 \xi) \frac{T}{2 a}+(1 \pm 4 \xi) \frac{\pi}{a^{2}} e^{-\pi / T a} \\
T a \ll 1, \quad x=a / 2,
\end{gathered}
$$


respectively. Now, whereas Eq. (89) is a conjecture, Eq. (88) is not [see Eq. (71)].

In order to address the regime $T a \gg 1$, instead of considering the prescription associated with Eq. (87), one can start again with Eq. (63), using Eq. (82) in Eq. (68), to show that $\left\langle T_{t t}\right\rangle$ is given by Eq. (83) with the term linear in $T / a$ missing. Therefore, it follows that

$$
\begin{aligned}
&\left\langle T_{t t}\right\rangle= \pm \frac{\xi}{2 \pi x^{2}}+(1 \mp 4 \xi)\left[\frac{\pi}{6} T^{2}-4 \pi T^{2} e^{-4 \pi T a}\right], \\
& T a \gg 1, \quad x / a \ll 1,
\end{aligned}
$$

and that

$$
\left\langle T_{t t}\right\rangle=\frac{\pi}{6} T^{2} \pm 16 \pi \xi T^{2} e^{-2 \pi T a}, \quad T a \gg 1, \quad x=a / 2 .
$$

The remark just after Eq. (84) applies here as well. That is, the "Casimir" vacuum energy density in Eq. (88) and the "blackbody" energy density in Eq. (90) play similar roles, but now it is the former that is shifted by a linear term in $T / a$. Recall that $\left\langle T_{x x}\right\rangle$ follows immediately from Eq. (77).

\section{B. Local thermodynamic stability}

Using the same setup as in Sec. II B, i.e., a small segment of the reflecting box where the temperature inside $\left(T_{\text {in }}\right)$ is slightly different from the temperature outside $\left(T_{\text {out }}\right)$, the following investigation of the regimes $T a \ll 1$ and $T a \gg 1$ will show that not all values of the coupling parameter $\xi$ are consistent with local thermodynamic stability. As the stress $\left\langle T_{x x}\right\rangle$ will be needed in Eq. (43), it is worth recalling once more that it can be obtained from the corresponding $\left\langle T_{t t}\right\rangle$ by simply taking $\xi=0$ [see Eq. (77)].

\section{Summing first over $\boldsymbol{n}$}

Looking at $\left\langle T_{t t}\right\rangle$ in Eq. (79), which holds when $T a \ll 1$, it follows that Eq. (43) yields $\Phi>0$ if $T_{\text {in }}>T_{\text {out }}$, i.e., energy leaves the segment. Thus, to ensure that thermodynamic equilibrium is restored (in other words, to ensure that $T_{\text {in }}$ drops), one must have that $\partial_{T}\left\langle T_{t t}\right\rangle>0$ everywhere in the box, then [from Eq. (79)] we obtain the constraint

$$
-\frac{1}{4} \leq \xi \leq \frac{1}{4},
$$

regardless of the type of boundary condition (i.e., whether it is Dirichlet or Neumann). It should be remarked that Eq. (92) would also follow from Eqs. (80) and (81). It should also be pointed out that Eq. (92) includes the minimal and conformal couplings, i.e., $\xi=0$, but this is not always the case since, for example, in a higher number of dimensions when a single Dirichlet wall is present (see Ref. [20]), conformal coupling is allowed whereas minimal coupling is not.
Turning to $T a \gg 1$, one takes $\xi=0$ in Eq. (84) to obtain $\left\langle T_{x x}\right\rangle$, and again uses Eq. (43) to conclude that $\Phi>0$ if $T_{\text {in }}>T_{\text {out }}$. Thus, requiring that $\partial_{T}\left\langle T_{t t}\right\rangle>0$ in Eq. (84), it results that $\xi$ must be such that

$$
\text { Neumann: } \xi \geq-1 / 4, \quad \text { Dirichlet: } \xi \leq 1 / 4 \text {, }
$$

which are constraints that are consistent with (but less stringent than) Eq. (92). It is worth noting that since Eq. (85) is essentially the "blackbody" energy density it does not set any constraint on the coupling parameter $\xi$.

\section{Summing first over $\boldsymbol{m}$}

Taking into account Eqs. (88) and (43), it follows that $\Phi>0$ if $T_{\text {in }}>T_{\text {out }}$. By requiring $\partial_{T}\left\langle T_{t t}\right\rangle>0$ in Eq. (88), it leads again to ${ }^{7}$ Eq. (93), instead of the more stringent constraint in Eq. (92). Clearly, the same outcome follows from Eq. (89).

Turning now to Eq. (90), an identical analysis again yields Eq. (93), whereas Eq. (91) sets no constraint on $\xi$. Note that Eq. (93) includes the minimal and conformal couplings in the constraints associated with both boundary conditions.

\section{Thermodynamics}

Perhaps some of the most interesting aspects of the ambiguities addressed in this paper are in the thermodynamics of the scalar radiation in an interval with reflecting edges. In order to obtain the internal energy $U$ one still uses Eq. (49), but now (unlike in Sec. II C) $\left\langle T_{t t}\right\rangle$ in Eq. (63) is nonhomogeneous due to the presence of the terms $\left\langle T_{t t}\right\rangle_{\text {vacuum }}^{\text {wall }}$ and $\left\langle T_{t t}\right\rangle_{\text {mixed }}^{\text {wall }}$ when $\xi \neq 0$ [note Eqs. (65), (67), and (68)]. In fact, as mentioned previously [see Eq. (80) and the text after it], $\left\langle T_{t t}\right\rangle_{\text {vacuum }}^{\text {wall }}$ in Eq. (65) carries nonintegrable divergences, and its integration from $x=0$ to $x=a$ requires regularization, which when properly implemented yields a vanishing contribution [21]. Thus, by considering Eq. (63) in Eq. (49), the only nontrivial integration is

$$
\begin{aligned}
& \int_{0}^{a}\left\langle T_{t t}\right\rangle_{\text {mixed }}^{\text {wall }} d x=\mp \xi a T^{2} \int_{0}^{2} \mu(p, T a) d p, \\
& \int_{0}^{a}\left\langle T_{t t}\right\rangle_{\text {mixed }}^{\text {wall }} d x=\mp \xi a T^{2} \int_{0}^{2} \nu(p, T a) d p,
\end{aligned}
$$

corresponding to the ambiguity in Eqs. (67) and (68).

Regarding the integration of $\mu$ over $p$ in Eq. (94), by noticing that

$\int \frac{\cos (p \pi) \cosh (k \pi / q)-1}{[\cos (p \pi)-\cosh (k \pi / q)]^{2}} d p=\frac{1}{\pi \cosh (k \pi / q)-\cos (p \pi)}$,

\footnotetext{
${ }^{7} \mathrm{By}$ considering a single reflecting wall at $x=0$, the constraints in Eq. (93) are also required [20,24].
} 
it follows from Eq. (74) that

$$
\int_{0}^{2} \mu(p, q) d p=0
$$

and therefore the first integration in Eq. (94) gives a vanishing contribution to the internal energy $U$. It is worth remarking that a consistency check can be implemented by considering Eq. (78) which leads promptly to Eq. (95), as it should.

Regarding now the integration of $\nu$ over $p$ in Eq. (94), one uses Eq. (69) to write

$$
\int_{0}^{2} \nu(p, q) d p=2 \int_{0}^{1} \nu(p, q) d p .
$$

Looking at the expression for $\nu$ in Eq. (75), it is a simple matter to show that

$$
\int_{0}^{1}\left[\frac{\pi}{4 q^{2}} \csc ^{2}\left(\frac{p \pi}{2}\right)-\pi \operatorname{cosech}^{2}(p q \pi)\right] d p=\frac{1}{q} \operatorname{coth}(q \pi)
$$

and

$$
\begin{aligned}
& \int_{0}^{1} \operatorname{cosech}^{2}\{q \pi(2 k \pm p)\} d p \\
& \quad=\mp \frac{1}{q \pi}[\operatorname{coth}\{q \pi(2 k \pm 1)\}-\operatorname{coth}\{q \pi 2 k\}] .
\end{aligned}
$$

Then one uses Eq. (98) to integrate the series in Eq. (75), i.e.,

$$
\begin{aligned}
- & \pi \sum_{k=1}^{\infty} \int_{0}^{1}\left[\operatorname{cosech}^{2}\{q \pi(2 k-p)\}\right. \\
& \left.+\operatorname{cosech}^{2}\{q \pi(2 k+p)\}\right] d p \\
= & -\frac{1}{q} \sum_{k=1}^{\infty}[\operatorname{coth}\{q \pi(2 k-1)\}-\operatorname{coth}\{q \pi(2 k+1)\}] \\
= & \frac{1}{q}-\frac{1}{q} \operatorname{coth}(q \pi) .
\end{aligned}
$$

Finally, by adding Eqs. (97) and (99), Eq. (96) yields

$$
\int_{0}^{2} \nu(p, q) d p=\frac{2}{q}
$$

which should be compared with Eq. (95). A consistency check can be done using Eq. (82), by integrating $q \nu$ over $p$, and then taking $q \rightarrow \infty$, resulting 2 as in Eq. (100). It should also be noted that Eqs. (95) and (100) offer an opportunity to check the consistency of the conjecture in Eq. (72) [namely, by integrating both sides of Eq. (72)], with no surprises.
Finally, one uses Eqs. (95) and (100) in the expressions in Eq. (94) to obtain the following contrasting contributions:

$\int_{0}^{a}\left\langle T_{t t}\right\rangle_{\text {mixed }}^{\text {wall }} d x=0, \quad \int_{0}^{a}\left\langle T_{t t}\right\rangle_{\text {mixed }}^{\text {wall }} d x=\mp 2 \xi T$,

respectively.

In order to obtain $U$ in Eq. (49), one goes back to Eq. (63), recalling that $\left\langle T_{t t}\right\rangle_{\text {vall }}^{\text {wall }}$ does not contribute with $U$. Then, taking into account Eqs. (23), (65), (66), and (101) gives $U$ in Eq. (6) (corresponding to "summing first over $n$ ") or $U$ in Eq. (7) (corresponding to "summing first over $m$ "). Note that Eqs. (6) and (7) differ from each other by the term

$$
(1 \mp 4 \xi) \frac{T}{2},
$$

which is consistent with the difference of the corresponding energy densities in Eq. (87).

Certain features of the ambiguity in Eqs. (6) and (7) were addressed in Sec. I. For example, it was mentioned that Eq. (6) is the result found in the literature [18,19], and that Eq. (7) violates the classical result that the internal energy $U$ should not depend on $\xi$ in the background considered here [21]. Proceeding as in Sec. II C, the following analysis concerns thermodynamic aspects of this ambiguity when $T a \ll 1$ and $T a \gg 1$.

\section{Summing first over $n$}

It can be quickly checked that Eq. (6) is obtained from Eq. (2), which applies to the circle, by replacing $a$ with $2 a$ and halving the final expression. In fact, the quantities $U$, $F$, and $S$ here can be obtained from those in Sec. II C 1 using this prescription. It results then that the thermodynamics of the scalar radiation in an interval with reflecting edges, according to "summing first over $n$," is the same as that discussed in Sec. II C 1. In particular, the third law of thermodynamics is also satisfied.

By integrating $\left\langle T_{\mu}^{\mu}\right\rangle$ over the box [note Eqs. (63) and (64)], due to the first integration in Eq. (101), it results that $U-\left\langle T_{x x}\right\rangle a=0$. As $\left\langle T_{x x}\right\rangle$ is the thermodynamic pressure $p$, the equation of state in Eq. (52) holds here as well.

\section{Summing first over $\boldsymbol{m}$}

When $\xi=0$ (minimal and conformal couplings), it is seen that Eq. (7) is also obtained from Eq. (4) by replacing $a$ by $2 a$ and halving the final expression. The quantities $U$, $F$, and $S$ result from the corresponding quantities in Sec. II C 2 by using this same prescription. Then, when $\xi=0$, the thermodynamics of the scalar radiation in an interval with reflecting edges, according to "summing first over $m$," closely resembles that in Sec. II C 2, including the violation of the third law. 
However, when $\xi \neq 0$, new issues arise. As mentioned above, one can obtain $U$ here by adding the term in Eq. (102) to the corresponding expression for $U$ in Sec. III C 1 . Beginning with the regime $T a \ll 1$, it results that

$U(T, a)=-\frac{\pi}{24 a}+(1 \mp 4 \xi) \frac{T}{2}+\frac{\pi}{a} e^{-\pi / T a}, \quad T a \ll 1$.

The internal energy in Eq. (103), which holds for low temperatures or small boxes, offers a good opportunity to confront the local thermodynamic stability in Sec. III B 2 with the global one, as remarked in Sec. I. Since global thermodynamic stability requires a positive heat capacity, i.e., $C=(1 \mp 4 \xi) / 2+\cdots>0$, one ends up consistently with the bounds on $\xi$ in Eq. (93) [see text in Sec. III B 2].

At this point, it is worth noting that by integrating $\left\langle T^{\mu}{ }_{\mu}\right\rangle$ over the box again, but now using the second integration in Eq. (101), we obtain the equation of state

$$
U-\mathrm{p} a=\mp 2 \xi T
$$

instead of that in Eq. (52). It should be remarked that $p$ in Eq. (77) does not depend on $\xi$.

Considering now Eqs. (103) and (104), we can integrate Eq. (5) and introduce a positive length scale $\ell$ that cannot depend on either $T$ or $a$, i.e.,

$$
\begin{gathered}
F(T, a)=-\frac{\pi}{24 a}-(1 \mp 4 \xi) \frac{T}{2} \ln (2 T \ell)-\frac{T}{2} \ln \frac{a}{\ell}-T e^{-\pi / T a}, \\
T a \ll 1 .
\end{gathered}
$$

It should be pointed out that $\ell$ arises only if $\xi \neq 0$. The entropy corresponding to Eq. (105) is given by

$$
\begin{aligned}
S(T, a)= & \ln \sqrt{\frac{a}{\ell}}+\frac{1}{2}(1 \mp 4 \xi)[\ln (2 T \ell)+1] \\
& +\left(\frac{\pi}{T a}+1\right) e^{-\pi / T a}, \quad T a \ll 1,
\end{aligned}
$$

which remains finite as $T \rightarrow 0$ for $\xi=1 / 4$ and $\xi=-1 / 4$ in the case of Dirichlet and Neumann boundary conditions, respectively, i.e., $S(T \rightarrow 0, a)=\ln \sqrt{a / \ell}$. Since $a / \ell$ is not a "universal constant," the third law of thermodynamics is still violated. ${ }^{8}$

Considering now the regime $T a \gg 1$, by adding Eq. (102) to the corresponding expression for $U$ in Sec. III C 1, one ends up with

$U(T, a)=\frac{\pi}{6} a T^{2} \mp 2 \xi T-4 \pi a T^{2} e^{-4 \pi T a}, \quad T a \gg 1$.

At this point, a remark that makes connection with an earlier paper is in order. By working with a single reflecting wall in

\footnotetext{
${ }^{8}$ However, it is worth remarking that according to some authors this is not a fault [34].
}

Ref. [24], we arrived at $U=\pi a T^{2} / 6 \mp \xi T$ which, when compared with Eq. (107), suggests that the factor of 2 in $\mp 2 \xi T$ is due to the presence of a second wall (as was conjectured in Ref. [24]). Note that in Ref. [24] only the summation over the "thermal" number $m$ appeared and thus the ambiguity of "summing first over $n$ " versus "summing first over $m$ " is not apparent.

By taking into account Eq. (104), one sees that $p$ is given by dropping the term $\mp 2 \xi T$ in Eq. (107) and dividing the resulting expression by $a$. The corresponding free energy is given by

$$
F(T, a)=-\frac{\pi}{6} a T^{2} \pm 2 \xi T \ln (2 T \ell)-T e^{-4 \pi T a}, \quad T a \gg 1
$$

where the length scale $\ell$ arises again [see Eq. (105)]. Finally, the behavior of the entropy at high temperatures or for large boxes follows from Eq. (108), namely,

$$
\begin{aligned}
S(T, a)= & \frac{\pi}{3} a T \mp 2 \xi[\ln (2 T \ell)+1]-(4 \pi T a-1) e^{-4 \pi T a}, \\
& T a \gg 1 .
\end{aligned}
$$

\section{FURTHER DISCUSSION}

In this paper we investigated the finite temperature $\left\langle T_{\mu \nu}\right\rangle$ of a massless scalar field on a circle and in an interval with reflecting edges (with Dirichlet or Neumann boundary conditions at the edges). In so doing, it was shown that $\left\langle T_{\mu \nu}\right\rangle$ involves double series which-due to the number of dimensions of the spacetime under consideration (i.e., $N=2$ )-are not absolutely convergent and that this fact is connected with ambiguities in the calculation of $\left\langle T_{\mu \nu}\right\rangle$. Namely, the order in which the two summations are evaluated leads to different results: summing first over the "boundary" number $n$, versus summing first over the "thermal" number $m$. By studying the associated thermodynamics of the contrasting expressions for $\left\langle T_{\mu \nu}\right\rangle$, it was found that in the case of the circle the ambiguity corresponds to the classic debate in the literature of whether or not zero modes should be ignored in the computation of partition functions. In the case of the interval with reflecting edges, one of the (nonhomogeneous) contrasting expressions for $\left\langle T_{\mu \nu}\right\rangle$ leads to the thermodynamics reported in the literature (obtained by using the partition function) whose internal energy $U$ does not depend on the curvature coupling parameter $\xi$ (as one would expect from a classical calculation), whereas the other expression for $\left\langle T_{\mu \nu}\right\rangle$ leads to a $U$ that does depend on $\xi$, which is rather unexpected.

It was shown that the ambiguities reported in this paper are nicely connected with classic results on infinite series which go back to the works of Ramanujan. In this context, 
a conjecture was presented whose consistency was checked in various instances.

Regarding the asymptotic regimes $T a \ll 1$ and $T a \gg 1$, although the ambiguities only affect subleading contributions in the contrasting expressions for $\left\langle T_{\mu \nu}\right\rangle$, their thermodynamic consequences are substantial. For example, "summing first over $m$ " leads to a violation of the third law of thermodynamics and, in the case of the interval with reflecting edges, also to an internal energy $U$ that depends on $\xi$, as just mentioned. It should be recalled that "summing first over $n$ " is not free of issues either. In the case of the circle, it leads to a $U$ that spoils the derivation of the CardyVerlinde formula (see Sec. I).

In the case of the interval with reflecting edges, the requirement of local thermodynamic stability led to different ranges of permissible values for $\xi$ corresponding to "summing first over $n$ " (where the constraint is more stringent and is the same for Dirichlet and Neumann boundary conditions) and to "summing first over $m$ " (where the constraint is less stringent and depends on the type of reflecting boundary condition). The "summing first over $m$ " side of the ambiguity allowed us to confront local and global thermodynamic stability, and the consistency of the constraints over the values of $\xi$ was verified.

Before closing, it is pertinent to raise an issue that may have already come to mind. As is typical of series that are not absolutely convergent, each way of summing the series may lead to different results and, consequently, to different physics. In light of this argument, one might question the relevance of the particular ways of evaluating the summations discussed in this paper, namely, "summing first over $n$ " versus "summing first over $m$." Whereas indeed other ways of evaluating the summations can lead to new thermodynamics (whose features may be interesting), those considered in this paper are closely connected with matters that have been addressed previously in the literature, in various contexts, as shown throughout the text.

This paper followed a line of investigation that was established long ago by Brown, Maclay, Dowker, and others, which consists in "deriving" blackbody thermodynamics from $\left\langle T_{\mu \nu}\right\rangle$. We intend to pursue this approach further in order to address classic issues that appear when event horizons are present in the background.

\section{ACKNOWLEDGMENTS}

We wish to thank Lucas dos Santos, Luis Fernando Mello, Marcia Kashimoto, and Claudemir de Oliveira for helpful conversations on the convergence of double series. This work is partially supported by "Fundação de Amparo à Pesquisa do Estado de Minas Gerais" (FAPEMIG) and "Coordenação de Aperfeiçoamento de Pessoal de Nível Superior" (CAPES).

\section{APPENDIX: THERMAL GREEN FUNCTIONS}

Consider a cavity in an $\mathrm{N}$-dimensional flat spacetime,

$$
d s^{2}=d t^{2}-d x^{2}-d y^{2}-d z^{2}-\cdots .
$$

One of the walls of the cavity coincides with the plane $x=0$ and another with $x=a>0$. The other walls, when they exist, are at infinity. A neutral scalar field $\phi$ with mass $M$ is in the cavity at temperature $T$. Then, the coordinate $x_{0}:=i t$ is taken to be real with period $\beta=1 / T$ (see, e.g., Ref. [35]). Considering further $x_{1}:=x, x_{2}:=y, x_{3}:=z$, Eq. (A1) becomes

$$
d s^{2}=-d x_{0}^{2}-d x_{1}^{2}-d x_{2}^{2}-d x_{3}^{2}-\cdots-d x_{N-1}^{2},
$$

and the boundary condition

$\phi\left(x_{0}, x_{1}, x_{2}, \ldots, x_{N-1}\right)=\phi\left(x_{0}+\beta, x_{1}, x_{2}, \ldots, x_{N-1}\right)$

must be observed. The (Euclidean) Green function satisfies [4]

$$
\left(\square_{\mathrm{x}}+M^{2}\right) G_{E}\left(\mathrm{x}, \mathrm{x}^{\prime}\right)=\delta\left(\mathrm{x}-\mathrm{x}^{\prime}\right),
$$

where $\square_{\mathrm{x}}:=-\partial_{0}^{2}-\partial_{1}^{2}-\partial_{2}^{2}-\partial_{3}^{2}-\cdots-\partial_{N-1}^{2}$.

\section{Periodic boundary condition}

The eigenfunctions of $\square_{\mathrm{x}}+M^{2}$ that satisfy Eq. (A3) and the periodic boundary condition, i.e.,

$$
\psi\left(x_{0}, x_{1}, x_{2}, \ldots, x_{N-1}\right)=\psi\left(x_{0}, x_{1}+a, x_{2}, \ldots, x_{N-1}\right),
$$

are given by

$\psi_{k}(\mathrm{x})=\eta \exp \left[i\left(k_{0} x_{0}+k_{1} x_{1}+\cdots+k_{N-1} x_{N-1}\right)\right]$,

where $\eta, k_{0}=2 \pi m / \beta, k_{1}=2 \pi n / a, \cdots, k_{N-1}$ are constants, and $m$ and $n$ are integers. The corresponding eigenvalues are

$$
E_{k}=k_{0}^{2}+k_{1}^{2}+\cdots+k_{N-1}^{2}+M^{2} .
$$

The constant $\eta$ in Eq. (A5) is set such that the Green function in Eq. (A4) is given by ${ }^{9}$

$$
\begin{aligned}
G_{E}\left(\mathrm{x}, \mathrm{x}^{\prime}\right)= & i \sum_{m=-\infty}^{\infty} \sum_{n=-\infty}^{\infty} \int_{0}^{\infty} d \tau \int_{-\infty}^{\infty} d k_{2} \cdots \\
& \int_{-\infty}^{\infty} d k_{N-1} e^{-i \tau E_{k}} \psi_{k}(\mathrm{x}) \psi_{k}^{*}\left(\mathrm{x}^{\prime}\right),
\end{aligned}
$$

\footnotetext{
${ }^{9}$ The expression in Eq. (A7) is known as Schwinger's "proper time" representation of the finite-temperature Green function (see, e.g., Ref. [36])
} 
and $M^{2}$ is taken to have an infinitesimal imaginary part to make the integration over $\tau$ in Eq. (A7) converge [37]. Then, it follows that

$$
\begin{aligned}
\left(\square_{\mathrm{x}}\right. & \left.+M^{2}\right) G_{E}\left(\mathrm{x}, \mathrm{x}^{\prime}\right) \\
= & -\sum_{m=-\infty}^{\infty} \sum_{n=-\infty}^{\infty} \int_{-\infty}^{\infty} d k_{2} \cdots \int_{-\infty}^{\infty} d k_{N-1} \psi_{k}(\mathrm{x}) \psi_{k}^{*}\left(\mathrm{x}^{\prime}\right) \\
& \times \int_{0}^{\infty} d \tau \frac{d}{d \tau} e^{-i \tau E_{k}},
\end{aligned}
$$

where the integration over $\tau$ yields simply minus unity. Now, by choosing

$$
|\eta|^{2}=\frac{(2 \pi)^{2-N}}{\beta a}
$$

and recalling the usual representation of the $\delta$ function, as well as Poisson's formula

$$
\sum_{l=-\infty}^{\infty} \delta(\lambda-2 \pi l)=\frac{1}{2 \pi} \sum_{l=-\infty}^{\infty} e^{-i l \lambda}
$$

it results that the right-hand side of Eq. (A8) is indeed $\delta\left(\mathrm{x}-\mathrm{x}^{\prime}\right)$, as Eq. (A4) requires.

A more workable expression for $G_{E}\left(\mathrm{x}, \mathrm{x}^{\prime}\right)$ in Eq. (A7) can be obtained. Noting Eq. (A5), two factors arise in Eq. (A7) that can be conveniently manipulated as follows:

$$
\begin{aligned}
& \sum_{l=-\infty}^{\infty} e^{-i \tau\left(4 \pi^{2} l^{2} / p^{2}\right)+i(2 \pi l / p) \Delta} \\
& \quad=\sum_{l=-\infty}^{\infty} \int_{-\infty}^{\infty} d \lambda \delta(\lambda-2 \pi l) e^{-i \tau\left(\lambda^{2} / p^{2}\right)+i(\lambda / p) \Delta} \\
& \quad=\frac{1}{2 \pi} \sum_{l=-\infty}^{\infty} \int_{-\infty}^{\infty} d \lambda e^{-i \tau\left(\lambda^{2} / p^{2}\right)+i(\lambda / p)(\Delta-l p)}
\end{aligned}
$$

where Eq. (A10) has been used in the last step. All of the integrations can now be performed [38], leading to

$$
\begin{aligned}
& G_{E}\left(\mathrm{x}, \mathrm{x}^{\prime}\right) \\
& =\frac{1}{(2 \pi)^{N / 2}} M^{\frac{N-2}{2}} \sum_{m=-\infty}^{\infty} \sum_{n=-\infty}^{\infty}\left(-\sigma^{(m, n)}\right)^{\frac{2-N}{4}} K_{\frac{N-2}{2}}\left(M \sqrt{-\sigma^{(m, n)}}\right),
\end{aligned}
$$

where [noting the coordinates in Eq. (A1)]

$$
\begin{aligned}
\sigma^{(m, n)}:= & \left(t-t^{\prime}-i m \beta\right)^{2}-\left(x-x^{\prime}-n a\right)^{2}-\left(y-y^{\prime}\right)^{2} \\
& -\left(z-z^{\prime}\right)^{2}-\cdots,
\end{aligned}
$$

and $K_{\nu}(\mathrm{z})$ is the modified Bessel function of the second kind.
Before addressing Dirichlet and Neumann boundary conditions, it should be mentioned that the Green function in Eq. (A12) is closely related to the thermal Hadamard function in Eq. (2.26) of Ref. [39], where certain aspects of a charged scalar field were investigated in a background with an arbitrary number of compact dimensions.

\section{Dirichlet and Neumann boundary conditions}

Considering now the Dirichlet boundary condition, i.e.,

$\psi\left(x_{0}, x_{1}=0, x_{2}, \ldots, x_{N-1}\right)=\psi\left(x_{0}, x_{1}=a, x_{2}, \ldots, x_{N-1}\right)=0$,

the eigenfunctions of $\square_{\mathrm{x}}+M^{2}$, which also satisfy Eq. (A3), are now given by

$$
\psi_{k}(\mathrm{x})=\eta \sin \left(k_{1} x_{1}\right) \exp \left[i\left(k_{0} x_{0}+k_{2} x_{2}+\cdots+k_{N-1} x_{N-1}\right)\right],
$$

whose eigenvalues are those in Eq. (A6), where $k_{0}=2 \pi m / \beta$ and $k_{1}=n \pi / a$, where $m$ and $n$ are integers as before. By using Eq. (A14) in Eq. (A7), noting Eqs. (A9) and (A10), the usual representation of the $\delta$ function and the Fourier sine series [25],

$$
\delta\left(x-x^{\prime}\right)=\frac{1}{a} \sum_{n=-\infty}^{\infty} \sin (n \pi x / a) \sin \left(n \pi x^{\prime} / a\right),
$$

it follows that Eq. (A8) becomes Eq. (A4), as it should. One now expands the sine functions in exponentials and manipulates the sums in Eq. (A7) as in Eq. (A11). The last step is to evaluate the integrations [38], resulting in

$$
\begin{aligned}
& G_{E}\left(\mathrm{x}, \mathrm{x}^{\prime}\right) \\
& =\frac{1}{(2 \pi)^{N / 2}} M^{\frac{N-2}{2}} \sum_{m=-\infty}^{\infty} \sum_{n=-\infty}^{\infty}\left[\left(-\sigma_{-}^{(m, n)}\right)^{\frac{2-N}{4}} K_{\frac{N-2}{2}}\left(M \sqrt{-\sigma_{-}^{(m, n)}}\right)\right. \\
& \left.-\left(-\sigma_{+}^{(m, n)}\right)^{\frac{2-N}{4}} K_{\frac{N-2}{2}}\left(M \sqrt{-\sigma_{+}^{(m, n)}}\right)\right],
\end{aligned}
$$

where

$$
\begin{aligned}
\sigma_{ \pm}^{(m, n)}:= & \left(t-t^{\prime}-i m \beta\right)^{2}-\left(x \pm x^{\prime}-2 n a\right)^{2} \\
& -\left(y-y^{\prime}\right)^{2}-\left(z-z^{\prime}\right)^{2}-\cdots .
\end{aligned}
$$

At this point, it should be remarked that the term $n=0$ in Eq. (A16) consistently reproduces the corresponding Green function in Ref. [32] (i.e., as $a \rightarrow \infty$ ). By setting $M \rightarrow 0$ and $N=4$ in Eq. (A16), the Green function in Ref. [16] is also consistently reproduced. ${ }^{10}$

\footnotetext{
${ }^{10}$ By setting $M \rightarrow 0$ and $N=4$ in Eq. (A16) it should also match the results in Ref. [4]; however, a typo was detected in Eq. (4.38) of Ref. [4]: "an" should be replaced by "2an."
} 
Turning now to the Neumann boundary condition, i.e.,

$$
\begin{aligned}
& \frac{\partial}{\partial x_{1}} \psi\left(x_{0}, x_{1}=0, x_{2}, \ldots, x_{N-1}\right) \\
& \quad=\frac{\partial}{\partial x_{1}} \psi\left(x_{0}, x_{1}=a, x_{2}, \ldots, x_{N-1}\right)=0,
\end{aligned}
$$

one proceeds as in the case of the Dirichlet boundary condition above, but now replacing the sine functions in Eqs. (A14) and (A15) with cosine functions. This gives a Green function that is still given by Eq. (A16), but with the minus sign between the terms containing Bessel functions replaced by a plus sign.
[1] J. D. Bekenstein, Black holes and entropy, Phys. Rev. D 7, 2333 (1973).

[2] S. W. Hawking, Particle creation by black holes, Commun. Math. Phys. 43, 199 (1975).

[3] L. Susskind and J. Lindesay, An Introduction to Black Holes, Information and The String Theory Revolution: The Holographic Universe (World Scientific, Singapore, 2005).

[4] N. D. Birrel and P. C. W. Davies, Quantum Fields in Curved Space (Cambridge University Press, Cambridge, England, 1982).

[5] K. Huang, Statistical Mechanics (John Wiley \& Sons, New York, 1987).

[6] J. S. Dowker, Effect of zero modes in statistical mechanics, Phys. Rev. D 37, 558 (1988).

[7] I. Brevik, K. A. Milton, and S. D. Odintsov, Entropy bounds in $R \times S^{3}$ geometries, Ann. Phys. (N.Y.) 302, 120 (2002).

[8] J. S. Dowker, Zero modes, entropy bounds and partition functions, Classical Quantum Gravity 20, L105 (2003).

[9] E. Elizalde and A. C. Tort, Thermal energy of a scalar field in a one-dimensional compact space, Phys. Rev. D 66, 045033 (2002).

[10] D. Kutasov and F. Larsen, Partition sums and entropy bounds in weakly coupled CFT, J. High Energy Phys. 01 (2001) 001.

[11] D. Klemm, A. C. Petkou, and G. Siopsis, Entropy bounds, monotonicity properties and scaling in CFTs, Nucl. Phys. B601, 380 (2001).

[12] Y. K. Yazdi, Zero modes and entanglement entropy, J. High Energy Phys. 04 (2017) 140.

[13] L. S. Brown and G. J. Maclay, Vacuum stress between conducting plates: An image solution, Phys. Rev. 184, 1272 (1969).

[14] J. S. Dowker and R. Critchley, Vacuum stress tensor in an Einstein universe: Finite-temperature effects, Phys. Rev. D 15, 1484 (1977).

[15] M. B. Altaie and J. S. Dowker, Spinor fields in an Einstein universe: Finite-temperature effects, Phys. Rev. D 18, 3557 (1978).

[16] S. Tadaki and S. Takagi, Casimir effect at finite temperature, Prog. Theor. Phys. 75, 262 (1986).

[17] W. Rudin, Principles of Mathematical Analysis (McGrawHill, Singapore, 1976).

[18] J. Ambjørn and S. Wolfram, Properties of the vacuum. I. Mechanical and thermodynamic, Ann. Phys. (N.Y.) 147, 1 (1983).
[19] S. C. Lim and L. P. Teo, Finite-temperature Casimir effect in piston geometry and its classical limit, Eur. Phys. J. C 60, 323 (2009).

[20] V. A. De Lorenci, L. G. Gomes, and E. S. Moreira, Jr., Hot scalar radiation setting bounds on the curvature coupling parameter, Classical Quantum Gravity 32, 085002 (2015).

[21] S. A. Fulling, Vacuum energy as spectral geometry, SIGMA 3, 094 (2007).

[22] H. B. Callen, Thermodynamics and an Introduction to Thermostatistics (John Wiley \& Sons, New York, 1985).

[23] S. A. Fulling, Aspects of Quantum Field Theory in Curved Space-Time (Cambridge University Press, Cambridge, England, 1989).

[24] E. S. Moreira, Jr., Thermodynamics of the scalar radiation in the presence of a reflecting plane wall, Braz. J. Phys. 49, 449 (2019).

[25] G. Arfken, Mathematical Methods for Physicists (Academic Press, New York, 1985).

[26] A. P. Prudnikov, Yu. A. Brychkov, and O. I. Marichev, Integrals and Series (Gordon and Breach, Amsterdam, 1986), Vol. 2.

[27] Wolfram Research, Inc., Mathematica, Version 11.2, Champaign, IL (2017).

[28] J. S. Dowker and K. Kirsten, Elliptic functions and temperature inversion symmetry on spheres, Nucl. Phys. B638, 405 (2002).

[29] Z. Mo and J. Jia, Generalized Schlömilch formulas and thermal Casimir effect of a fermionic rectangular box, Phys. Rev. A 98, 012512 (2018).

[30] F. Ravndal and D. Tollefsen, Temperature inversion symmetry in the Casimir effect, Phys. Rev. D 40, 4191 (1989).

[31] E. S. Moreira, Jr., Hot scalar radiation around a cosmic string setting bounds on the coupling parameter $\xi$, J. High Energy Phys. 03 (2017) 105.

[32] V. A. De Lorenci, L. G. Gomes, and E. S. Moreira, Jr., Local thermal behaviour of a massive scalar field near a reflecting wall, J. High Energy Phys. 03 (2015) 096.

[33] A. A. Saharian, The generalized Abel-Plana formula with applications to Bessel functions and Casimir effect, arXiv:0708.1187.

[34] R. M. Wald, "Nernst theorem" and black hole thermodynamics, Phys. Rev. D 56, 6467 (1997).

[35] S. A. Fulling and S. N. M. Ruijsenaars, Temperature, periodicity and horizons, Phys. Rep. 152, 135 (1987).

[36] D. Deutsch and P. Candelas, Boundary effects in quantum field theory, Phys. Rev. D 20, 3063 (1979). 
[37] B. S. DeWitt, Dynamical Theory of Groups and Fields (Blackie \& Son, London, 1965).

[38] I. S. Gradshteyn and I. M. Ryzhik, Table of Integrals, Series, and Products (Academic Press, New York, 2007).
[39] E. R. Bezerra de Mello and A. A. Saharian, Finite temperature current densities and Bose-Einstein condensation in topologically nontrivial spaces, Phys. Rev. D 87, 045015 (2013). 